\title{
Ecological niche of Cryptococcus neoformans var. grubii and Cryptococcus gattii in decaying wood of trunk hollows of living trees in Jabalpur City of Central India
}

\author{
N. Grover · Shesh Rao Nawange · J. Naidu • \\ S. M. Singh · Archana Sharma
}

Published online: 21 April 2009

(C) Springer Science+Business Media B.V. 2009

\section{Erratum to: Mycopathologia (2007) 164:159-170 DOI 10.1007/s11046-007-9039-2}

We would like to make following corrections to our article that appeared in Mycopathologia (2007) 164:159-170.

Abstract, page 159, column 1, line 12, "two varieties" should read "two species".

Abstract, page 159, column 1, lines 13-15, " $C$. gattii and C. $n$. var. grubii isolates belonged to serotype B and serotype A, respectively" should read "C. gattii isolates belonged to serotype B".

Abstract, page 159, column 2, line 4, "both varieties" should read "both species".

The online version of the original article can be found under doi:10.1007/s11046-007-9039-2.

N. Grover · S. R. Nawange - S. M. Singh · A. Sharma

Medical Mycology Research Laboratory,

Department of Biological Science,

Rani Durgavati University,

Jabalpur, MP 482001, India

J. Naidu

Department of Zoology and Biotechnology,

Govt. Model Autonomous Science College,

Jabalpur, MP 482001, India

S. M. Singh $(\bowtie)$

Flat 2 C, Pavitra Apartment, South Civil lines,

Jabalpur, MP 482001, India

e-mail: smsingh_rdvv@rediffmail.com
Abstract, page 159, column 2, line 9, "this yeast" should read "these pathogens".

Introduction, page 159, column 2, line 4-5, " $C$. neoformans has been subdivided into three varieties and five serotypes" should read " $C$. neoformans, earlier subdivided into three varieties and five serotypes, is now known to contain $C$. neoformans var. grubii (serotype A), C. neoformans var. neoformans (serotype D) and a distinct species C. gattii (serotype B and C)".

Introduction, page 160, column 1, line 24, "harbor one or both $C$. neoformans varieties" should read "harbor C. neoformans and/or C. gattii".

Materials and methods, page 161, column 2, line 8, "the presence of $C$. neoformans" should read "the presence of C. neoformans and C. gattii".

Materials and methods, column 1, page 161, line 13, "the varieties" should read "the species".

Materials and methods, column 1, page 161, line 19, "the varieties" should read "the species".

Results, page 162, column 2, line 1, "rectangular leaves" should read "oblong leaves".

Results, page 162, column 2, lines 3-17 should be read with revised data in Table 1.

Discussion, page 165, line 6, "varieties" should read "species".

Discussion, page 165, line 11 "varieties" should read "species".

Discussion, page 168, column 1, lines 2-3, “C. n. var. neoformans serotype $\mathrm{D}$ and $\mathrm{AD}$ " should read " $C$. $n$. var. neoformans serotype D". 
Discussion, page 168, column 2, line 24, "ecological niche of $C$. neoformans" should read "ecological niche of C. gattii".

Table 1, Title, "isolation of Cryptococcus neoformans" should read "isolation of C. neoformans var. grubii and C. gattii".

Table 1, S. no. 5, corrected version should read as "Syzygium cumini (a) Bark 01/50 (50\%) under column $C$. gattii and 02/50 (4\%) under column
C. $n$.var. grubii (b) Hollow debris 07/50(14\%) under column C.gattii and 01/50 (2\%) under column C. n.var. grubii. Total frequency $11 / 50=22 \%$ ".

Table 1, "Ohms ratio" should read "Odds ratio". Figure 1, Legend, "varieties and serotype of $C$. neoformans" should read "C. neoformans and C. gattii".

Figure 4, Legend, "C. neoformans" should read "C. neoformans and C. gattii". 